\title{
Comportamento da variação paleosecular durante o período de transição do Paleozoico-Mesozoico
}

Felipe Freitas (ON/MCTI)*, Daniel Ribeiro Franco (ON/MCTI)

Copyright 2016, SBGf - Sociedade Brasileira de Geofísica

Este texto foi preparado para a apresentação no VII Simpósio Brasileiro de Geofísica. Ouro Preto, 25 a 27 de outubro de 2016. Seu conteúdo foi revisado pelo Comitê Ouro Preto, 25 a 27 de outubro de 2016. Seu conteúdo foi revisado pelo Comitê de seus associados. É proibida a reprodução total ou parcial deste material para propósitos comerciais sem prévia autorização da SBGf.

\section{Resumo}

Neste trabalho avaliamos em caráter preliminar o comportamento da dispersão de um dado conjunto de polos geomagnéticos virtuais (PGV's) como função das paleolatitudes, visando uma correlação com 0 comportamento do campo magnético terrestre após um período considerado como de grande estabilidade do Geodínamo (Superchron Kiaman - Irving e Parry, 1963; Opdyke, 1995; Driscoll \& Evans, 2016). Esses PGV's correspondem ao período conhecido como Hiperzona de Polaridade Mista do Permotriássico (HPMPT) ou Illawarra (265 - 245) Ma; Os dados são oriundos da literatura, apresentam cobertura inter-hemisférica e foram obtidos de forma cuidadosa e sistemática para atender aos critérios de qualidade aceitos como confiáveis pela comunidade científica internacional, por exemplo critérios de Van der Voo (e.g., Van der Voo, 1990).

\section{Introdução}

O campo magnético terrestre ou campo geomagnético (CG), apresenta variações em seu comportamento direcional e em sua intensidade que vem sendo motivo de debate durante muitos anos. A discussão sobre sua origem causou tamanha inquietação que chegou a ser apontada como um dos cinco mais importantes problemas da Física por Albert Einsten, há cerca de cem anos atrás (Courtillot \& Le Mouel, 2007). Dentre os fenômenos que mais despertam interesse acerca do comportamento do geodínamo, estão as chamadas reversões de polaridade magnética e os Superchrons (estados geodinâmicos singulares e que duram períodos de tempo maiores que 20Ma - Jacobs, 2001) cujas hipóteses apresentadas ainda são controversas (Kruiver, 2000). Atualmente vem sendo debatida na literatura algumas hipóteses, dentre as quais a de que - baseado na teoria do dipolo geocêntrico axial (DGA) - durante episódios em que o campo apresenta uma alta taxa de reversões, a componente dipolar do CG estaria operando sob um regime de mais baixa energia do que durante os períodos dos Superchrons, e que consequente sua energia estaria dissociada, por exemplo, nas demais componentes quadrupolares e octupolares obtidas na expansão em harmônicos esféricos gerada a partir do modelo G publicado em 1998 por McFadden em colaboração com outros pesquisadores (McFadden et al, 1998). Assim, se considerarmos esta hipótese como válida, são esperados valores de dispersão de PGV's em função da paleolatitude mais elevados durante episódios de alta taxa reversões magnéticas (em que o campo estaria menos dipolar), do que durante episódios de estabilidade magnética (em que o campo estaria mais dipolar). Neste intuito, o presente trabalho consistiu em avaliar em caráter preliminar tais valores de dispersão de dados paleomagnéticos para o intervalo conhecido como Illawarra (aproximadamente 265Ma a 245Ma - período de alta taxa de reversões e subsequente a um período notavelmente mais estável e bem reportado), atendendo a abrangência em cobertura inter-hemisférica.

\section{Metodologia}

Com o intuito de se avaliar o padrão de dispersão de PGV's como função da paleolatitude, e o padrão da variação paleosecular VPS durante a (HPMPT), desenvolveu-se um algoritmo que utiliza os dados magnéticos de inclinação e declinação da magnetização remanente característica (MRC) - proveniente da magnetização adquirida pelas rochas e sedimentos amostrados, durante a fase de sua formação em sitio - e as coordenadas dos locais de amostragem. A partir dos dados de entrada são calculados os parâmetros estatísticos: declinação e inclinação média, latitude e longitude do polo paleomagnético, paleolatitude, os parâmetros de dispersão $\left(S, S_{L}\right.$ e $\left.S_{u}\right)$, e os parâmetros estatísticos do limite de confiabilidade.

Os dados utilizados como input para os cálculos acima dados provenientes da literatura e que atendam aos critérios de qualidade aceitos como confiáveis pela comunidade científica internacional - são préselecionados de acordo com uma metodologia cuidadosa, seguindo uma lista de recomendações que garantem a melhor aproximação possível dos valores reais procurados. Tais critérios abrangem a idade dos estudos, a metodologia empregada, a idade das amostras utilizadas pelo autor e a necessidade de dados direcionais de MRC confiáveis.

\section{Resultados}

A partir dos dados gerados pelo programa desenvolvido pelos pesquisadores do Observatório Nacional (ON $\mathrm{MCTI}$ ) e os colaboradores do - Instituto de Astronomia, Geofísica e Ciências Atmosféricas (IAG-USP); Universidade da Califórnia (Santa Cruz); e instituto de Física da UERJ (IF-UERJ) - obtivemos o gráfico da dispersão dos polos geomagnéticos virtuais como função das paleolatitudes, com cobertura inter-hemisférica e intervalo enquadrado dentro do limite de transição do período Permiano para o Triássico, aproximadamente (265 a 245)Ma. Os resultados foram sobrepostos às curvas obtidas pelo modelo proposto em (McFadden, 
1991), Veja Figura 1, com o intuito de encontrar uma similaridade ao o modelo.

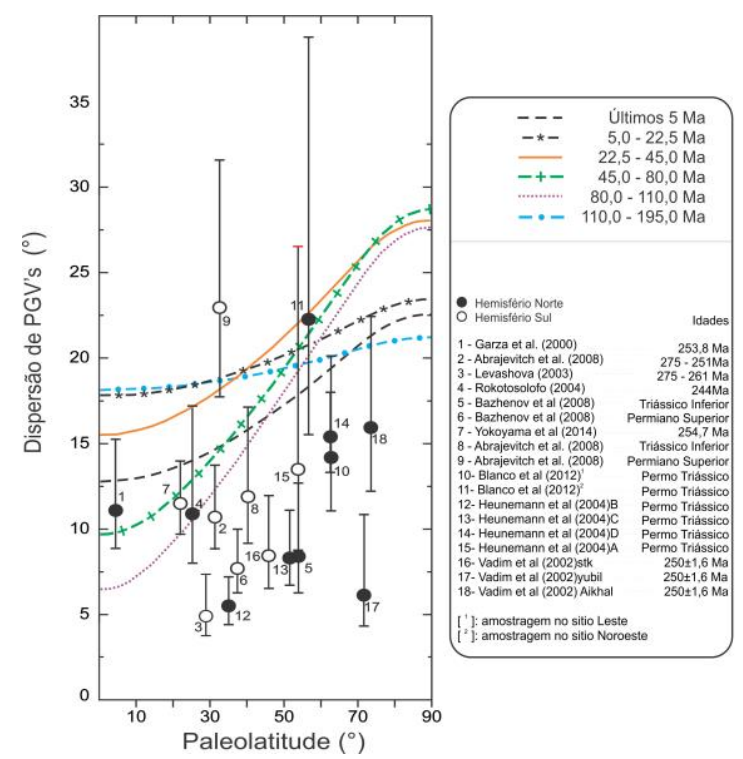

Figura 1: curvas obtidas pelo modelo sugerido em (McFadden et al, 1991); dispersão de PGV's como função das paleolatitudes (este estudo).

\section{Discussão e Conclusões}

Embora a quantidade de informações paleomagnéticas com qualidade suficientemente boa para proporcionar uma discussão definitiva a respeito do comportamento da variação paleosecular (VPS) e do funcionamento do geodínamo durante episódios em que o campo apresentou uma alta taxa de reversões ainda não seja elevada, uma análise preliminar do parâmetro (S) da dispersão dos polos geomagnéticos virtuais (PGV's) como função das paleolatitudes, sugere valores de dispersão mais baixos para o intervalo que compreende a Hiperzona de Polaridade Mista do Permotriássico do que para os demais períodos de tempo estudados a partir das curvas obtidas pelo Modelo G (McFadden et al, 1998). É possível sugerir ainda uma baixa dependência dos valores do $S$ com os valores de paleolatitudes, tal comportamento é similarmente observado na curva correspondente ao período Jurássico-Cretáceo (195 110)Ma e na curva obtida para o Paleoceno (5,0 a $22,5) \mathrm{Ma}$ - ver também a figura 2 . Em ambos os casos observa-se um resultado preliminar que corrobora a premissa adotada pelo modelo, isto é, durante períodos de grande instabilidade geomagnética - menores contribuições da componente dipolar e maiores contribuições das componentes quadrupolares e octopolares - acompanhados de pouca dependência entre a dispersão de PGV's com as paleolatitudes.

\section{Agradecimentos}

Felipe Freitas agradece ao Conselho Nacional de Desenvolvimento Científico e Tecnológico (CNPq) pelo provimento e manutenção da bolsa de mestrado.

\section{Referências}

Courtillot, V., Le Mouël, J.-L., 2007. The study of earth's magnetism (1269-1950): a foundation by peregrinus and subsequent development of geomagnetism and paleomagnetism. American Geophysical Union, Reviews of Geophysics, 45, RG3008 / 2007, Paper number 2006RG000198.

Driscoll P.E., Evans D. A. D., 2016. Frequency of Proterozoic geomagnetic superchrons. Earth and Planetary Science Letters, 437: 9-14.

JACOBS, J., 2001. The cause of superchrons. Astron. Geophys., 42, 6: 30-31.

Kruiver P.P., Dekkers M. J., Langereis C.G., 2000. Secular variation in Permian red beds from Dôme de Barrot, SE France. Earth and Planetary Science Letters Elsevier, 179: 205-217.

McFadden, P.L. Merrill, R.T. McElhinny, M.W., 1998. Dipole/quadrupole family modeling of paleosecular variation. Journal of Geophysical Research: Solid Earth (1978-2012), vol 93, n. B10: 11583-11588.

McFadden, P.L. Merrill, R.T. McElhinny, M.W. Lee. S., 1991 Reversals of the Earth's Magnetic Field and Temporal Variations of the Dynamo Families. Journal of Geophysical Research, vol 96, n. B3: 3923-3933.

Opdyke, N.D., 1995. Magnetostratigraphy of PermianCarboniferous time. Em: Geochronology Time Scales and Global Stratigraphic Correlation, eds. Berggren, W.A., Kent,D.V. Aubry, M.-P. Hardenbol, J. Soc. Sed. Geol. Spec. Publ. (SEPM), Special Publications, 54: 41-47.

Van der voo, R., 1990. The reliability of paleomagnetic data. Tectonophysics, vol. 184, n. 1: 1-9. 


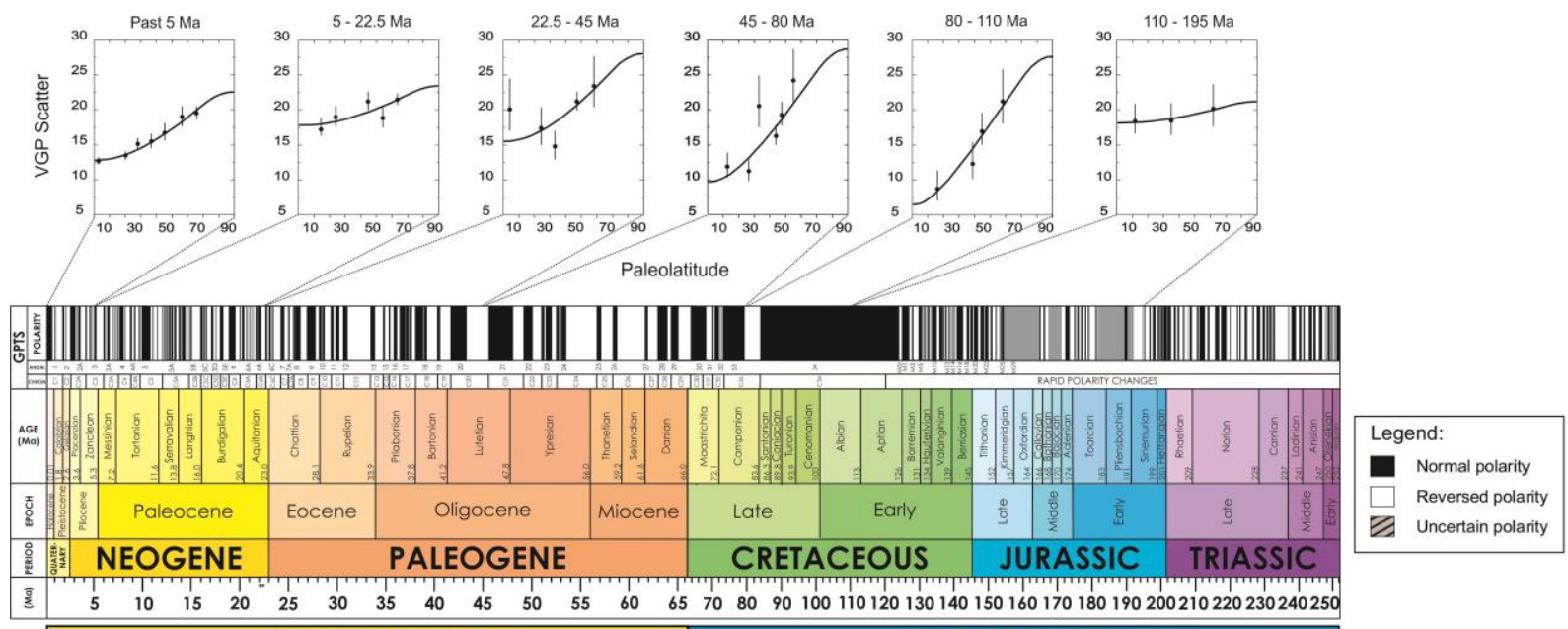

\section{CENOZOIC $\quad$ MESOZOIC}

Figura 2 - Escala temporal de polaridade geomagnética. Modificado de (McFadden, 1991). 